\title{
Seroprevalence of toxoplasmosis among reproductive-aged women in Myanmar and evaluation of luciferase immunoprecipitation system assay
}

Khin Myo Aye ${ }^{1,2}$, Eiji Nagayasu ${ }^{2 *}$, Myat Htut Nyunt ${ }^{1}$, Ni Ni Zaw ${ }^{1}$, Kyaw Zin Thant ${ }^{1}$, Myat Phone Kyaw ${ }^{1}$ and Haruhiko Maruyama

\begin{abstract}
Backgrounds: Primary infection with Toxoplasma gondii during pregnancy can pose serious health problems for the fetus. However, the epidemiological status of toxoplasmosis among reproductive-aged population in Myanmar is largely unknown. Although luciferase immunoprecipitation system (LIPS) assays for serodiagnosis of toxoplasmosis was developed mostly using mouse infection model, had not been tested by using field-derived human samples.

Methods: A total of 251 serum samples were collected from reproductive-aged women, residing in Shwegyin township, Bago region, Myanmar and analyzed with a commercial ELISA kit, as well as in-house LIPS assays.

Results: The overall seroprevalence for Toxoplasma gondii infection by the commercial ELISA was $11.5 \%$. No clear risk factor was identified except for being in the younger age group (15-30 years old). Overall, LIPS assays showed low sensitivity when the commercial ELSA was used as a reference test.

Conclusion: We identified the epidemiological situation of toxoplasmosis in some rural communities in Myanmar. The data obtained here will serve as a primary information for the effort to reduce toxoplasmosis in this region. Although looked promising in the previous experiments with mouse infection model, we found that the reported LIPS procedures need further improvements to increase the sensitivities.
\end{abstract}

Keywords: Toxoplasma gondii, Luciferase immunoprecipitation system, Nanoluciferase, Recombinant antigen, Enzyme-linked immunoassay, Myanmar

\section{Background}

Toxoplasmosis is a parasitic disease caused by Toxoplasma gondii. Between 30 and $50 \%$ of the global human population is thought to be infected by this protozoan pathogen [1]. Humans can be infected through accidental ingestion of oocysts excreted from infected cats, or by eating raw or undercooked meat containing tissue cysts [2]. Acute

\footnotetext{
* Correspondence: enagayas@med.miyazaki-u.ac.jp

${ }^{2}$ Division of Parasitology, Department of Infectious Diseases, Faculty of Medicine, University of Miyazaki, Kiyotake, Japan

Full list of author information is available at the end of the article
}

toxoplasmosis in most cases is asymptomatic and $T$. gondii can persist in host tissues by forming bradyzoites. In AIDS patients, Toxoplasma encephalitis is one of the most common brain lesions, which is lethal if left untreated [3]. Primary infection in pregnant women with $T$. gondii can lead to congenital toxoplasmosis [4]. Based on a recent metaanalysis, it was estimated that the transmission rates of Toxoplasma infection from acutely infected pregnant women to fetus are 5, 13 and 32\%, if exposure was in 1st, 2nd or 3rd trimester, respectively [5]. If congenital transmission is confirmed, treatment with anti-parasitic drugs

(C) The Author(s). 2020 Open Access This article is licensed under a Creative Commons Attribution 4.0 International License, which permits use, sharing, adaptation, distribution and reproduction in any medium or format, as long as you give appropriate credit to the original author(s) and the source, provide a link to the Creative Commons licence, and indicate if changes were made. The images or other third party material in this article are included in the article's Creative Commons licence, unless indicated otherwise in a credit line to the material. If material is not included in the article's Creative Commons licence and your intended use is not permitted by statutory regulation or exceeds the permitted use, you will need to obtain permission directly from the copyright holder. To view a copy of this licence, visit http://creativecommons.org/licenses/by/4.0/ The Creative Commons Public Domain Dedication waiver (http://creativecommons.org/publicdomain/zero/1.0/) applies to the data made available in this article, unless otherwise stated in a credit line to the data. 
should be initiated to prevent complications of congenital toxoplasmosis. Therefore, diagnosing Toxoplasma infection early and precisely is of crucial importance.

Until now, serological tests have been the main diagnostic methods for screening of toxoplasmosis. Serological test based on Sabin-Feldman dye test has been considered the gold standard because of its high sensitivity and specificity in humans. However, it requires live parasites and highly skilled personnel and therefore is usually performed only in reference laboratories [6]. Other tests, such as indirect fluorescent antibody test (IFAT), latex agglutination test (LAT), indirect haemagglutination (IHA), modified agglutination test (MAT), and enzyme-linked immunosorbent assay (ELIS A), are useful to screen T. gondii infection [7].

Luminescent assays are believed to be the most sensitive detection method due to the ability of signal multiplication and amplification [8]. Luciferase immunoprecipitation system (LIPS) is a relatively new luminescent assay technology, originally developed by Burbelo et al., that is based on liquid phase antibody binding event with luciferase-tagged antigens [9]. LIPS assay has been applied to a number of infectious diseases including strongyloidiasis [10], onchocerciasis [11], loiasis [12] and several autoimmune diseases such as type I diabetes, Sjogren's syndrome, and systemic lupus erythematous in humans and demonstrated superior performances over conventional ELISA [13].

Recently, T. gondii recombinant fusion proteins of Nluc (a luciferase enzyme) and diagnostic antigens (rGRA6, rGRA7, rGRA8 and rBAG1) were produced and evaluated for use in LIPS assay. The newly developed LIPS protocols with these fusion recombinant antigens were tested using sera collected from mice experimentally infected with $T$. gondii, and an international standard anti-Toxoplasma human serum, TOXM [14].

The aim of this study is twofold: firstly, to estimate the sero-epidemiological status and risk factors in reproductive-aged women, living in this study areas of Myanmar by using a widely recommended commercial ELISA kits; and secondary to acquire information regarding the performance of the LIPS assays compared to the commercial ELISA kits.

\section{Methods}

\section{Study population}

A cross-sectional descriptive study was carried out at five villages of the Shwegyin Township, Bago Region, Myanmar, from January to March, 2017. The minimal sample size required was estimated to be 246 with $5 \%$ precision and 95\% confidence levels, assuming a prevalence of $20 \%$ [15]. Thereafter, adult women within reproductive age (15-55 years), apparently healthy were included in this study. Women who showed signs and symptoms of severe infection were excluded from this study. Villages in
Shwegyin township (Fig. 1) were selected due to high population densities of cats and animals in their homes and surroundings. No documented data on seroprevalence of anti-T. gondii antibodies in female of reproductive age and associated risk factors were available at the time of the survey. Structured questionnaires (Table S1) were used to assess their knowledge and to explore the possible risk factors of toxoplasmosis in those population.

\section{Serum sample collection}

Venous blood $(1 \mathrm{~mL})$ was collected under aseptic condition. The blood was allowed to clot for at least 30 mins at room temperature. Serum was separated by centrifugation at $1500 \mathrm{~g}$ for $15 \mathrm{mins}$ and stored at $-20^{\circ} \mathrm{C}$ until analyzed.

\section{Serological investigations}

All the samples were measured for IgG antibodies against $T$. gondii by a commercial ELISA kit (Platelia ${ }^{\text {Tn }}$ Toxo-IgG, BioRad, Marnes-la-Coquette, France; hereinafter called "Platelia IgG-ELISA"), according to the manufacture's instruction. The positive samples by Platelia IgG-ELISA were tested again by Platelia ${ }^{\text {Tm }}$ Toxo-IgM (Bio-Rad, Marnes-la-Coquette, France). The same set of sera were tested with LIPS assays with four different recombinant $T$. gondii antigens (NlucrGRA6, -rGRA7, -rGRA8 and -rBAG1). For selected samples, additional testing was conducted by Architect Toxo-IgG (Abbott Laboratories, Abbott Park, IL, USA) at a clinical laboratory service provider, Rinsho Miyazaki, Japan.

The detailed procedures for LIPS assays can be found in our previous publication [14]. Briefly, the recombinant proteins were adjusted to the following concentrations by diluting the original stocks with buffer A $(50 \mathrm{mM}$ Tris $\mathrm{pH} 7.5,100 \mathrm{mM} \mathrm{NaCl}, 5 \mathrm{mM}$ $\mathrm{MgCl} 2$, 1\% Triton X-100); Nluc-rGRA6 (0.4 ng/ $\mu \mathrm{L})$, Nluc-rGRA7 $(1.5 \mathrm{ng} / \mu \mathrm{L})$, Nluc-rGRA8 $(1.0 \mathrm{ng} / \mu \mathrm{L})$ and Nluc-rBAG1 $(0.7 \mathrm{ng} / \mu \mathrm{L})$. Sera were diluted 10-fold with buffer $\mathrm{A}$ as well. The diluted recombinant protein $(50 \mu \mathrm{L})$, diluted serum $(10 \mu \mathrm{L})$ and buffer A $(50 \mu \mathrm{L})$ were mixed, and antibody binding to antigens were allowed to occur by incubating them at room temperature for $1 \mathrm{~h}$. Protein A/G beads were added to capture the antigen-antibody complex. These immune complexes were washed eight times with $200 \mu \mathrm{L}$ of buffer A and two times with $200 \mu \mathrm{L}$ of PBS, then furimazine substrate (Nano-Glo luciferase assay substrate [Promega, Madison, WI, USA]) was added. Luciferase activity deriving from the Nluc-T.gondii antigen fusion proteins bound to the beads were measured by a luminometer (DTX 800 multimode detector, Beckman Coulter, Tokyo, Japan) and expressed as relative light unit (RLU). The serum samples, positive and negative controls were tested in duplicate wells for each run.

Seroreactivities to Nluc-rBAG1 antigen was further evaluated by conventional ELISA. Wells of ELISA 


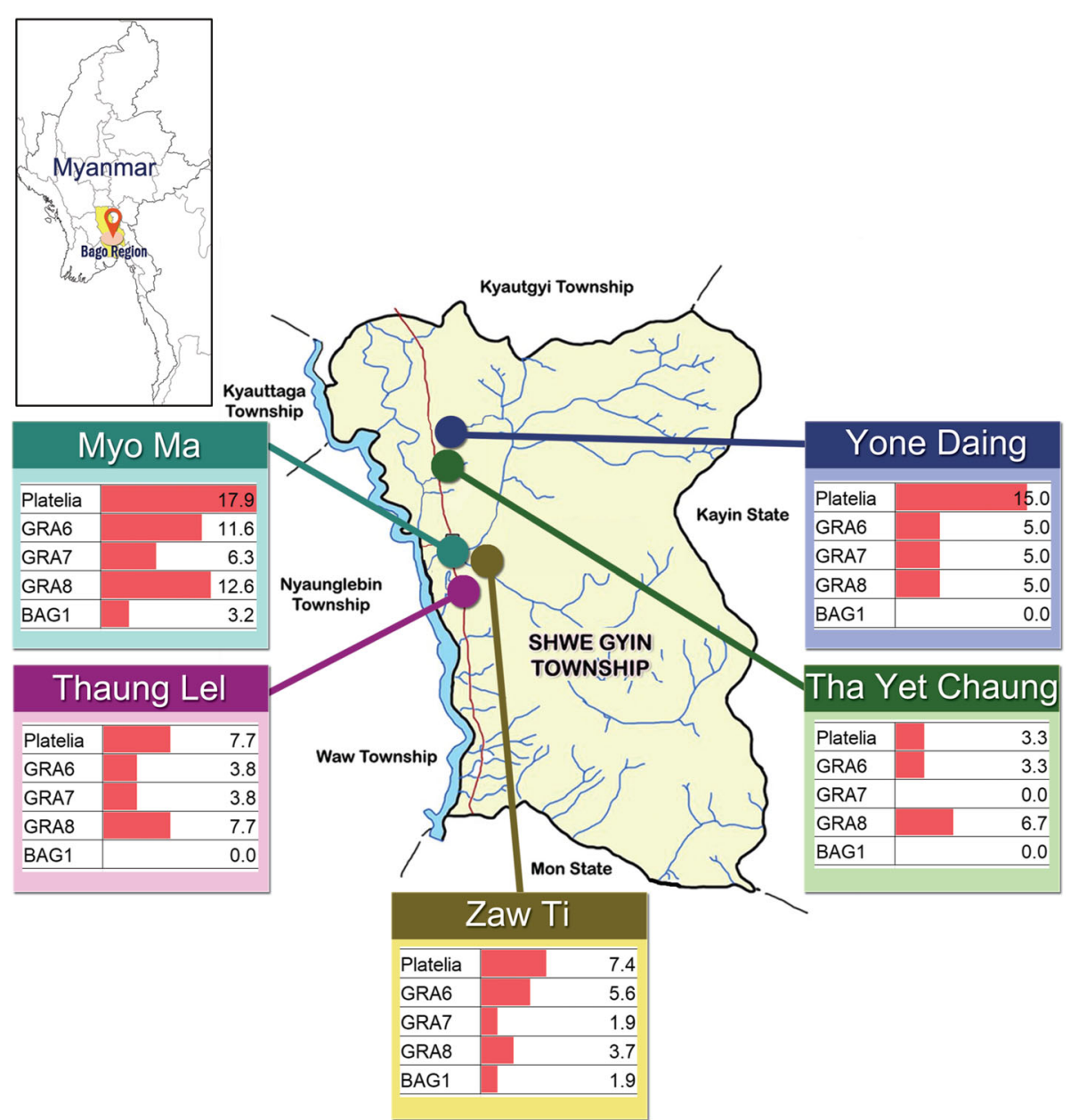

Fig. 1 Sampling locations and seroprevalences calculated for each location; Thaung Lel, Zaw Ti, Tha Yet Chaung, Yone Daing and Myo Ma belong to Shwegyin Township, Bago Region, Myanmar. The seroprevalences based on Platelia IgG-ELISA and LIPS assays with Nluc-rGRA6, -rGRA7, -rGRA8 and -rBAG1 are presented

plates (C96 Maxisorp Nunc-immunoplate; ThermoScientific, Roskilde, Denmark) were coated with $50 \mu \mathrm{L}$ of coating buffer $(50 \mathrm{mM}$ carbonate/bicarbonate $[\mathrm{pH}$ 9.6]) containing $5 \mu \mathrm{g} / \mathrm{mL}$ of Nluc-rBAG1, overnight at $4{ }^{\circ} \mathrm{C}$. By using a plate washer, AMW-8R (BioTec, Tokyo, Japan), the wells were washed with $300 \mu \mathrm{L}$ of washing buffer $(0.05 \%$ Tween 20-PBS) three times. The wells were then blocked with $150 \mu \mathrm{L}$ of blocking buffer $(1 \%[\mathrm{w} / \mathrm{v}]$ casein in TBS [Tris-buffered saline], $\mathrm{pH}$ 7.6) at room temperature for $2 \mathrm{~h}$. After removing the blocking buffer, $50 \mu \mathrm{L}$ of human sera diluted 1 : 100 with the blocking buffer were added and incubated at $37^{\circ} \mathrm{C}$ for $1 \mathrm{~h}$. The plates were washed with $300 \mu \mathrm{L}$ of washing buffer three times, then incubated with $50 \mu \mathrm{L}$ of secondary antibody (peroxidase-labeled polyclonal rabbit anti-human IgG, Dako, Glostrup, Denmark) at 1:2000 dilution in blocking buffer at $37^{\circ} \mathrm{C}$ for $1 \mathrm{~h}$. The wells were washed with $300 \mu \mathrm{L}$ of washing buffer three times, then $50 \mu \mathrm{L}$ of $\mathrm{ABTS}^{\mathrm{R}}$ peroxidase substrate (1-component; KPL, Gaithersburg, MD, USA) was added into the each well, and kept at room temperature for 30 mins. The plates were measured at $405 \mathrm{~nm}$ by $\mathrm{iMark}^{\mathrm{Tm}}$ microplate reader (Bio-Rad, Irvine, CA, USA). Each serum sample was run in duplicate wells. Positive and negative control were included in each run.

The same set of sera (i.e., 251 sera) were also tested with conventional ELISA using commercially available recombinant $T$. gondii P30 (SAG1) antigen (Bio-Rad, Irvine, CA, USA). All the procedures were the same as mentioned above except $2 \mu \mathrm{g} / \mathrm{mL}$ rSAG1 was used for coating each well.

\section{Statistical analysis}

Statistical analyses were performed by using SPSS version 22.0 (IBM Corp., New York, NY, USA). For the categorical data, Chi-square test or Fisher's exact test (when the minimum cell counts were less than 5) were 
used. Odds ratio (OR) with 95\% confidence interval (CI) were also calculated. Sensitivity, specificity, positive predictive value, negative predictive value and Cohen's Kappa value were calculated by SPSS. Correlation and Mann-Whitney $t$ tests were used to compare antibody titers in different groups. Differences were considered significant if $P<0.05$. Graphs were built using GraphPad Prism 7 (GraphPad Software Inc., San Diego, CA, USA).

\section{Results}

Demographic and behavioral profiles of the study population

The age of female included in this study ranged between 15 and 54 years (median age; 32 years). Among them, $72.5 \%(182 / 251)$ were married, $22.7 \%(57 / 251)$ had bad obstetric history and $64.1 \%(161 / 251)$ were employed. Regarding the contact with animals, 62.9\% (158/251) owned a cat (s) and $53.8 \%(135 / 251)$ had stray cat (s) at

Table 1 Bivariate analysis of selected demographic, environmental, behavioral variables with seroprevalence of Toxoplasma gondii infection based on Plateria IgG ELISA in (251 samples) reproductive-aged women

\begin{tabular}{|c|c|c|c|}
\hline Variable & Number of positive residents (\%) & Odds ratio $(95 \% \mathrm{Cl})$ & $p$ \\
\hline \multicolumn{4}{|l|}{ Age (years) } \\
\hline $15-30$ & 19 (16.8) & $2.56(1.14-5.88)$ & 0.02 \\
\hline$>30$ & $10(7.2)$ & & \\
\hline \multicolumn{4}{|l|}{ Occupation } \\
\hline Employed & $17(10.6)$ & & \\
\hline Unemployed & $12(13.3)$ & $1.3(0.59-2.87)$ & 0.51 \\
\hline \multicolumn{4}{|c|}{ Bad obstetric history } \\
\hline No & $14(11.2)$ & & \\
\hline Yes & $6(10.5)$ & $0.93(0.34-2.57)$ & 0.89 \\
\hline \multicolumn{4}{|c|}{ Contact with cat (s) or not } \\
\hline No & $6(10.9)$ & & \\
\hline Yes & $16(15.5)$ & $1.5(0.55-4.09)$ & 0.43 \\
\hline \multicolumn{4}{|c|}{ Presence of stray cat (s) at home } \\
\hline No & $15(12.9)$ & & \\
\hline Yes & $14(10.4)$ & $0.78(0.36-1.69)$ & 0.53 \\
\hline \multicolumn{4}{|c|}{ Presence of other animal (s) } \\
\hline No & $19(17.4)$ & & \\
\hline Yes & $10(7.0)$ & $0.36(0.16-0.81)$ & 0.01 \\
\hline \multicolumn{4}{|c|}{ Contact with other animal (s) } \\
\hline No & $4(4.5)$ & & \\
\hline Yes & $6(11.1)$ & $2.63(0.71-9.77)$ & 0.15 \\
\hline \multicolumn{4}{|c|}{ Drinking untreated water } \\
\hline No & $13(15.7)$ & & \\
\hline Yes & $16(9.5)$ & $0.57(0.26-1.24)$ & 0.16 \\
\hline \multicolumn{4}{|c|}{ Eating raw vegetables } \\
\hline No & $10(13.9)$ & & \\
\hline Yes & 19 (10.6) & $0.74(0.32-1.67)$ & 0.46 \\
\hline \multicolumn{4}{|c|}{ Eating undercooked meat } \\
\hline No & $27(11.0)$ & & \\
\hline Yes & $2(40.0)$ & $5.41(0.87-33.8)$ & 0.07 \\
\hline \multicolumn{4}{|l|}{ Contact with soil } \\
\hline No & $3(10.0)$ & & \\
\hline Yes & $26(11.8)$ & $1.2(0.34-4.24)$ & 0.78 \\
\hline
\end{tabular}

${ }^{*} P<0.05$ considered as significant. $95 \% \mathrm{Cl}=$ confidence interval

Groups that answered "No" were used as reference group for odds ratio calculation for yes/no questions. Groups of $>30$ years old or employed were used as reference group for the odds ratio analysis for age and occupation, respectively 
their home respectively. In addition, 56.6\% (142/251) answered other animals were present at their home or compounds. Total $71.3 \%$ (179/251) had a habit of eating raw vegetables after washing with water. Most of them $(98.0 \%$ [246/251]) cooked meat thoroughly. Few people (10.0\% [25/251]) live in their farms but $88.0 \%(221 / 251)$ had a history of contact with soil for gardening or cleaning their yards. Only $0.8 \%(2 / 251)$ had heard the word toxoplasmosis but most $72.5 \%$ (182/251) had awareness of disease transmission from cats through health workers, elder persons, friends, media such as radio, television and magazines.

\section{Seroprevalence and associated risk factors of Toxoplasma infection}

Based on the results obtained by Platelia IgG-ELISA, the overall seroprevalence for $T$. gondii infection in our study population was calculated to be $11.5 \%(29 / 251)$, varying from 3.3 to $17.9 \%$ (Fig. 1).

The seroprevalence judged by the Plateria Toxo-IgG ELISA and associated factors calculated by bivariate analysis are shown in (Table 1). Being in the younger group was associated with increased risk of having positive test result $(\mathrm{OR}=2.56 ; 95 \% \mathrm{CI}=1.14-5.88, P=0.02)$. Contact with cats or presence of stray cats was not proven to be risk factors in this study group. The presence of other animals in their home or surroundings were shown to be negatively associated with positive antibody test results by Platelia IgG-ELISA $(\mathrm{OR}=0.36$; 95\% $\mathrm{CI}=0.16-0.81, P=0.01)$.

\section{Evaluation of LIPS assays}

LIPS assays were performed on the same set of sera. Because absolute cut-off values for LIPS assays have not been established, tentative cut-offs were calculated using the mean + 3SD RLUs obtained from all the negative samples by Platelia IgG-ELISA $(n=222)$. Based on these, $7.2 \%$ (18/251), $4.0 \%$ (10/251), 8.4\% (21/251) and 1.6\% (4/ 251) of the samples were judged as positive by LIPS assay with Nluc-rGRA6-, GRA7-, GRA8- and BAG1LIPS assay, respectively. When Platelia IgG-ELISA was considered as a reference test, the sensitivities of LIPS assays were not high. In particular, none of the Platelia IgG-ELISA positive samples $(n=29)$ were positive with LIPS assay with Nluc-BAG1 (sensitivity $=0 \%)($ Table S2). When RLU values were compared between Platelia IgGELISA positive and negative groups, the positive group had significantly higher RLU values than the negative

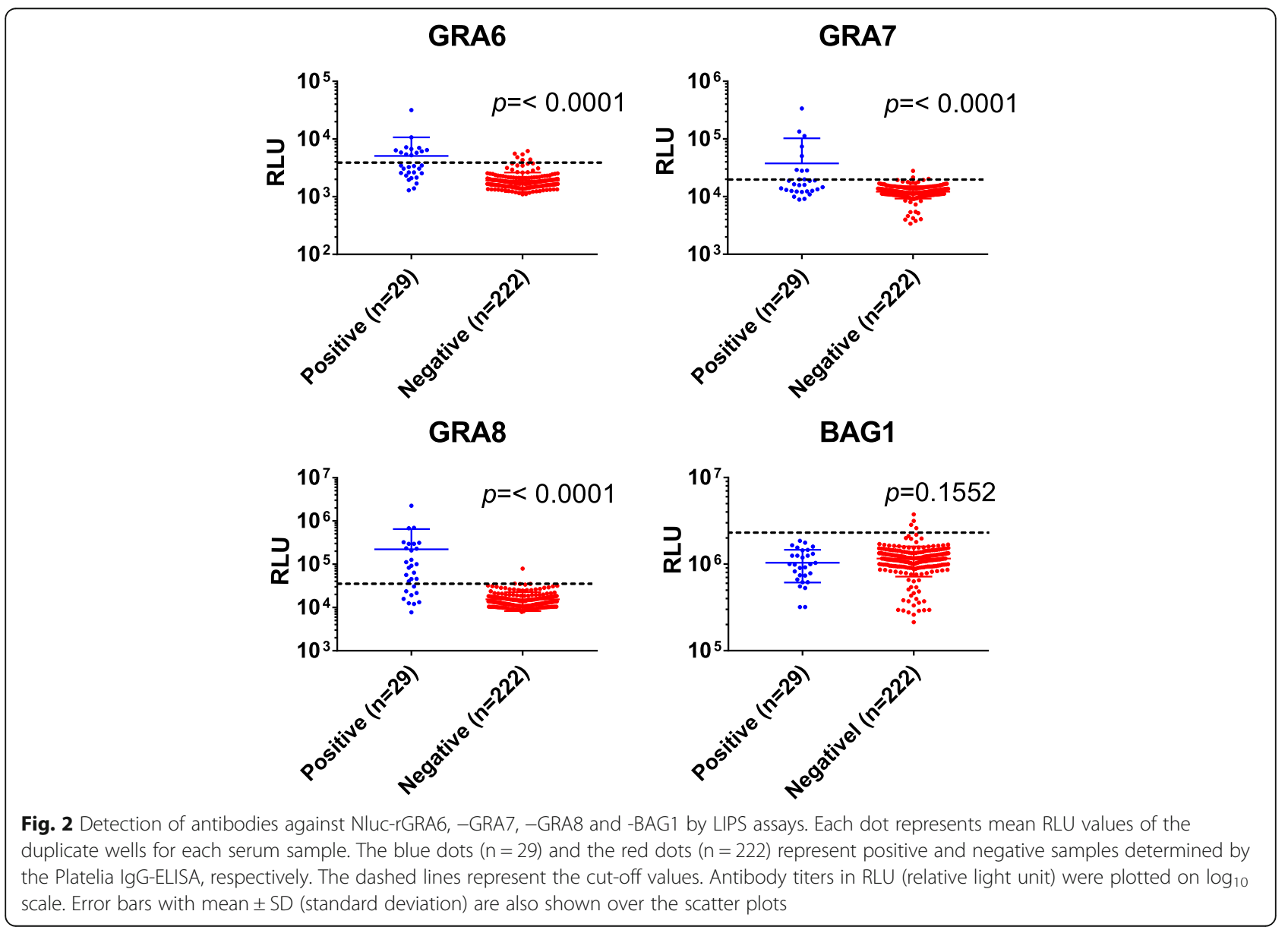


group for Nluc-rGRA6-, GRA7- and GRA8-LIPS assays as expected. On the other hand, no statistically significant difference was observed for BAG1-LIPS assay (Fig. 2).

A total of 40 samples exhibited positive results by at least one of the six assays (Platetlia IgG-ELISA, LIPS assays with four different antigens and rSAG1-ELISA). The remaining samples $(n=211)$ were negative by all tests. The serological pattern of these 40 positive sera is illustrated in Fig. 3. Identification numbers were assigned to each of these 40 sera (numbers 1-40). Eleven sera (serum numbers from 30 to 40 in Fig. 3) were negative by Platelia IgG-ELISA but positive by LIPS assays with one or two recombinant antigens and by rSAG1ELISA. None of the Nluc-rBAG1-LIPS positive sera were positive by Platelia IgG-ELISA (sample numbers 30, and 38-40).

Correlations between OD values obtained from Platelia IgG-ELISA and RLU values from LIPS assay were assessed. Moderate positive correlations were found in case of Platelia IgG-ELISA and GRA6-, GRA7- and GRA8-LIPS assays (Fig. 4). On the other hand, no

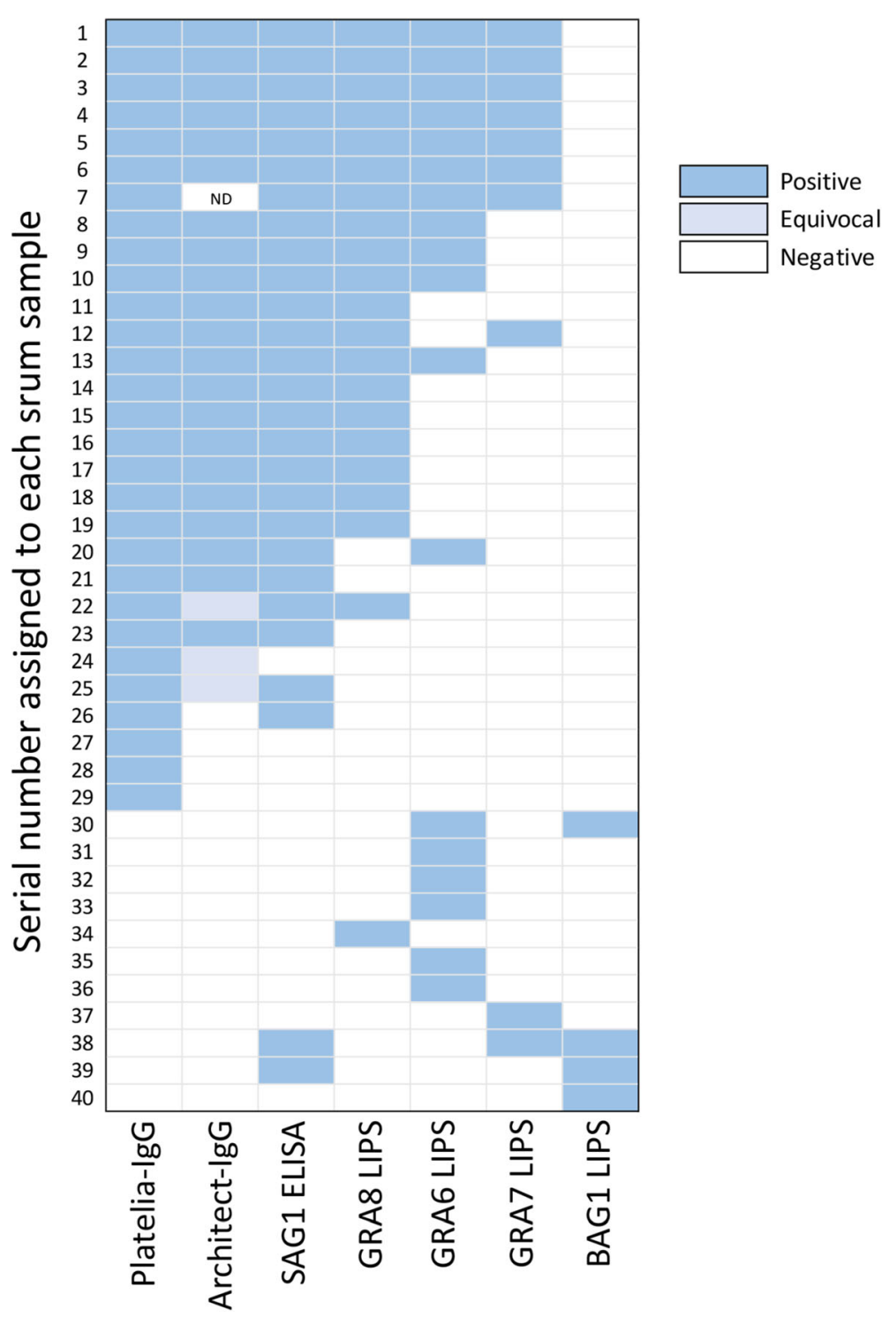

Fig. 3 Pattern of seroreactivities against T. gondii antigens evaluated by Platelia IgG-ELISA, LIPS assays with four different T. gondii antigens and commercially available rSAG1 by conventional ELISA. Each positive test result is shown as a rectangle filled in blue, while negative test results are shown as blank areas. Numbers arranged along the Y-axis (identification [ID] numbers 1-40) represent sample identification numbers arbitrary assigned to each serum sample. For example, serum ID "No. 1" showed positive test results by all tests except for the BAG1-LIPS assay, while the serum ID "No. 40" was positive only by the GRA6-LIPS assay Samples that were diagnosed as negative by all tests $(n=211)$ are not shown in this figure 

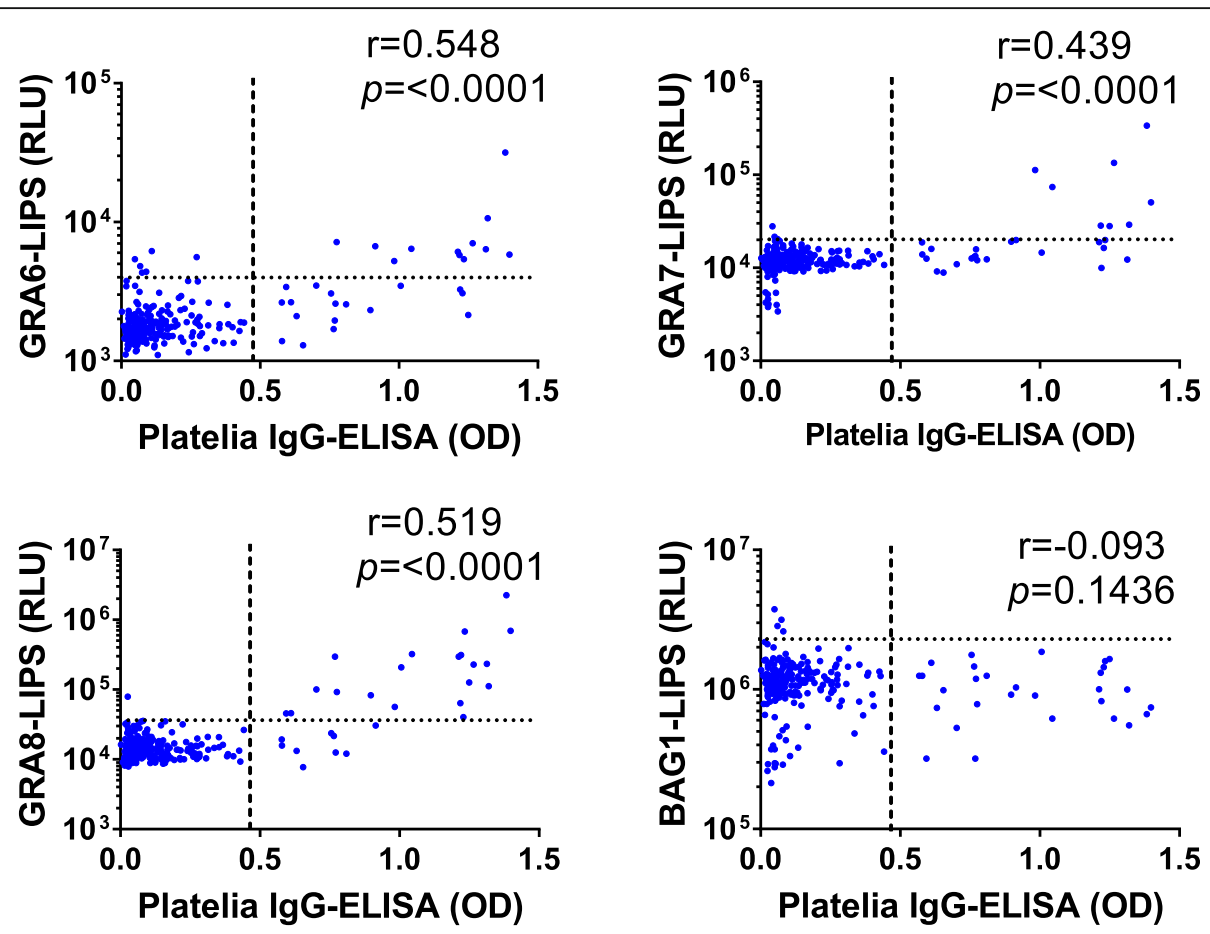

Fig. 4 Correlation between Platelia IgG-ELISA and LIPS assays. The ELISA OD (optical density) values are plotted on the $x$-axis, while the LIPS RLU values are plotted on the $y$-axis (in log10 scale). Each dot indicates mean values of duplicate wells for each sample. The dashed lines represent cut-off values. There is a moderate correlation between Platelia IgG-ELISA and Nluc-rGRA6-LIPS assay $(r=0.548, P<0.0001), r$ rGA8-LIPS assay ( $r=$ $0.519, P<0.0001)$; fair correlation with rGRA7-LIPS assay $(r=0.439, P<0.0001)$ respectively and statistically significant whereas there is no correlation between Platelia IgG-ELISA and Nluc-rBAG1-LIPS assay and statistically not significant $(r=-0.093, P=0.1436)$

correlation was found between Platelia IgG-ELISA and BAG1-LIPS assay. The RLU values obtained from NlucrBAG1-LIPS and OD values obtained from NlucrBAG1-ELISA showed a moderate correlation $(r=0.577$, $P<0.0001$ ) (Fig. S1).

Similarly to the results obtained by Nluc-rBAG1-LIPS (Fig. 2), none of the Platelia IgG-ELISA positive sera were judged as positive by Nluc-rBAG1-ELISA. Figure 5 shows the results of ELISA with rBAG1. There were five samples judged positive in this assay (five dots located above the cut-off line). All of these five positive samples by this rBAG1-ELISA belonged to the group of Platelia IgG-ELISA negative individuals (Fig. 5, shown as Control $[n=222]$ in the figure).

All serum samples were also evaluated with rSAG1ELISA (Platelia IgG-ELISA was considered as a reference test) and the seroprevalence was (27/251) $10.7 \%$ in which two samples were also positive by Nluc-rBAG1LIPS assay and rBAG1-ELISA.

Commercial Platelia IgG-ELISA positive sera and LIPS assay positive (ie. Platelia IgG-ELISA negative) were also assessed by Platelia ${ }^{\mathrm{Tm}}$ Toxo-IgM. Only one sample was positive and one was equivocal by Platelia ${ }^{\text {Tx }}$ Toxo-IgM respectively.

Given some degree of difference between test results obtained from Plateria IgG-ELSIA and LIPS assays, a

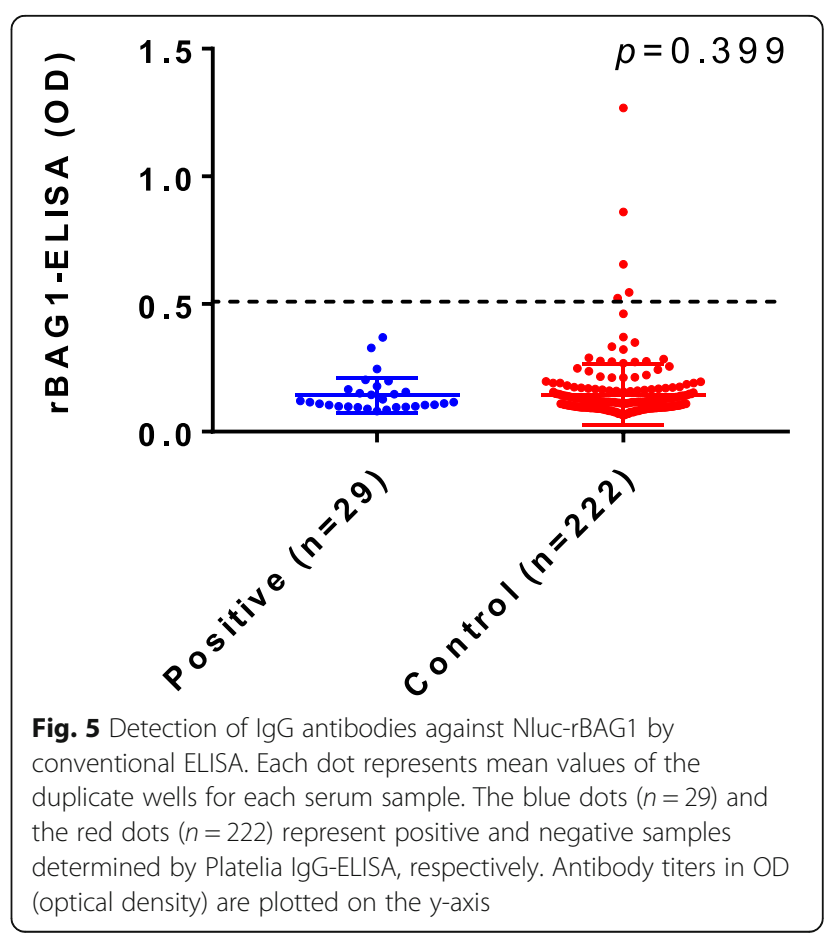


subset of samples were also tested with Architect IgG ELSIA. All the Plateria IgG-positive samples except four were diagnosed either as positive or equivocal by Architect IgG ELSIA (Fig. 3). All the randomly selected Plateria IgG ELISA negative samples $(n=20)$ were also negative by Architect IgG ELISA. The resultant kappa value was 0.260 , which was interpreted as fair agreement.

\section{Discussion}

Primary T. gondii infection in pregnant women can lead to several adverse outcomes such as miscarriage and fetal damage. In this study, 251 sera obtained from healthy reproductive-age women living in rural area of Myanmar were examined. The prevalence of toxoplasmosis based on Platelia IgG-ELISA was 11.5\% (29/251), which was lower than the seroprevalence reported previously in Myanmar, such as $23.5 \%$ among school children in Pyin Oo Lwin and Naung Cho [16], 31.7\% among refugee/migrant pregnant women from Myanmar [17] and $30.7 \%$ among pregnant women attending at the antenatal clinic at Yangon Central Hospital [15].

The younger (15-30 years old) was associated with higher chance of presenting Platelia IgG-ELISA positive test results. In general, improvement of overall sanitary conditions is expected to decrease the seropositivities in younger generations. However, such trend was not observed in this study. Counterintuitively, presence of other animal (s) was found to be negatively associated with the seropositivity. This may not be a direct association but rather a consequence of confounding, necessitating more investigation. Contact with cat is thought to be one of the risk factors contracting toxoplasmosis [18]. In this study, more percentage of the residents with contact with cats had positive Plateria IgG-ELISA results. However, the difference could not be proven to be statistically significant, which may due to the small sample size.

Using the same set of the sera, LIPS assays with four different recombinant antigens were assessed. Various degrees of discordance among tests were observed by LIPS assays with four different recombinant $T$. gondii antigens. While specificities and negative predictive values were high, sensitivities were low overall. Among LIPS assays with the four antigens, the assay with Nluc-rGRA8 exhibited the highest agreement with the Plateria IgG-ELISA test results. Even with that test, the sensitivity was only $68.97 \%$. Therefore, although initially looked promising based on the mouse experimental infection model, application of the reported LIPS protocol to human clinical samples require further improvements.

\section{Conclusions}

The overall seroprevalence for Toxoplasma gondii infection by the Plateria IgG-ELISA was $11.5 \%$ in this study population in reproductive-aged women, based on the Plateria IgG-ELISA. This data will serve as a primary information for the effort to reduce toxoplasmosis in this region. The current LIPS assay protocols with recombinant $T$. gondii antigens need improvements to increase their sensitivities.

\section{Supplementary Information}

Supplementary information accompanies this paper at https://doi.org/10. 1186/s12879-020-05650-y.

Additional file 1: Table S1. Seroprevalence of toxoplasmosis among reproductive-aged women in Myanmar measured by luciferase immunoprecipitation system assay.

Additional file 2: Table S2. Comparison of diagnostic performances of (inactivated T.gondii antigen) commercial ELISA kit (Platelia IgG-ELISA) and (Nluc-rGRA6, -rGRA7, -rGRA8 and -rBAG1) LIPS assay.

Additional file 3: Figure S1. Correlation between the seroreactivities against Nluc-rBAG1 evaluated by ELISA and LIPS assays. The OD (optical density) values obtained by ELISA and the RLU (relative light unit) values obtained by LIPS are plotted on the $x$ - and the $y$-axis, respectively. Each dot represents mean value of duplicate wells for each sample. There is a good correlation $(r=0.577)$ and statistically significant $(P<0.0001)$ between the two assays.

\section{Abbreviations}

Cl: Confidence interval; ELISA: Enzyme-linked immunosorbent assay; IgG: Immunoglobulin G; LIPS: Luciferase immunoprecipitation system; OD: Optical density; OR: Odds ratio; RLU: Relative light unit

\section{Acknowledgments}

Special thanks to the health authorities and volunteers from Shwegyin Township, Bago Region, Myanmar for their help throughout the survey and samples collection.

\section{Authors' contributions}

KMA performed the experiments, analyzed the data, and wrote the initial draft of the manuscript. KMA, MHN and NNZ collected the samples. KMA and MHN designed the study for field survey and analyzed the data. KZT, MPK and MHN supervised the field survey. EN designed the experimental procedures. EN and HM supervised the analysis and revised the manuscript. $H M, E N$, and KMA contributed to data interpretation and manuscript writing. The authors read and approved the final manuscript.

\section{Funding}

This work was supported by a grant from the Japanese Agency for Medical Research and Development (AMED, Emerging/Re-emerging Infectious

Diseases Project of Japan, grant no: 5fk0108046h0003) and by the Kurozumi Medical Foundation, Japan. The funders had no role in study design, data collection and analysis, decision to publish, or preparation of the manuscript.

\section{Availability of data and materials}

The datasets used and/or analysed during the current study available from the corresponding author on reasonable request.

\section{Ethics approval and consent to participate}

This study was conducted with the approval from the ethical review committee of Department of Medical Research (Myanmar) (ERC number: 000816), IRB number (IRB00008835) and this work was supported by the research grants number "419" from the Institutional Review Board (IRB), University of Miyazaki for recombinant experiments. Written informed consents were obtained from all subjects who were 18 years or older. When the participants were under 18 years old, written 
informed consent from parents or guardians as well as written assent from participants were obtained.

Information collected from each participant was kept confidential. Participation in this research was entirely voluntary and the participants had the right to withdraw from the research at any time.

\section{Consent for publication}

Not applicable.

\section{Competing interests}

The authors declare that they have no competing interests.

\section{Author details}

'Department of Medical Research, Ministry of Health and Sports, Yangon, Myanmar. ${ }^{2}$ Division of Parasitology, Department of Infectious Diseases, Faculty of Medicine, University of Miyazaki, Kiyotake, Japan.

Received: 14 July 2020 Accepted: 22 November 2020

Published online: 30 November 2020

\section{References}

1. Fernandez-Reyes D, Flegr J, Prandota J, Sovičková M, Israili ZH. Toxoplasmosis - a global threat. Correlation of latent toxoplasmosis with specific disease burden in a set of 88 countries. PLoS One. 2014;9:e90203.

2. Zemene E, Yewhalaw D, Abera S, Belay T, Samuel A, Zeynudin A. Seroprevalence of Toxoplasma gondii and associated risk factors among pregnant women in Jimma town, southwestern Ethiopia. BMC Infect Dis. 2012;12:337.

3. Weiss LM, Kim K. The development and biology of bradyzoites of Toxoplasma gondii. Front Biosci J Virtual Libr. 2000;5:D391-405.

4. Robert-Gangneux F, Dardé M-L. Epidemiology of and diagnostic strategies for toxoplasmosis. Clin Microbiol Rev. 2012;25:264-96.

5. Li XL, Wei HX, Zhang $H$, Peng HJ, Lindsay DS. A meta analysis on risks of adverse pregnancy outcomes in Toxoplasma gondii infection. PLoS One. 2014;9:e97775.

6. Udonsom R, Buddhirongawatr R, Sukthana Y. Is Sabin-Feldman dye test using T. gondii tachyzoites from animal inoculation still the best method for detecting Toxoplasma gondii antibodies? Southeast Asian J Trop Med Public Health. 2010;41:1059-64.

7. Wang Z, Ge W, Huang SY, Li J, Zhu XQ, Liu Q. Evaluation of recombinant granule antigens GRA1 and GRA7 for serodiagnosis of Toxoplasma gondii infection in dogs. BMC Vet Res. 2014;10:158.

8. Hall MP, Unch J, Binkowski BF, Valley MP, Butler BL, Wood MG, et al. Engineered luciferase reporter from a deep sea shrimp utilizing a novel imidazopyrazinone substrate. ACS Chem Biol. 2012;7:1848-57.

9. Burbelo PD, Ching KH, Bren KE, ladarola MJ. Searching for biomarkers: Humoral response profiling with luciferase immunoprecipitation systems (LIPS). Expert Rev Proteomics. 2011;8. https://doi.org/10.1586/epr.1511.1523.

10. Siddiqui AA, Bisoffi Z, Buonfrate D, Sequi M, Mejia R, Cimino RO, et al. Diagnostic accuracy of five serologic tests for Strongyloides stercoralis infection. PLoS Negl Trop Dis. 2014;8:e2640.

11. Burbelo PD, Leahy HP, ladarola MJ, Nutman TB. A four-antigen mixture for rapid assessment of Onchocerca volvulus infection. PLoS Negl Trop Dis. 2009;3:e438.

12. Burbelo PD, Ramanathan $R$, Klion AD, ladarola MJ, Nutman TB. Rapid, novel, specific, high-throughput assay for diagnosis of Loa loa infection. J Clin Microbiol. 2008:46:2298-304

13. Burbelo PD, Lebovitz EE, Notkins AL. Luciferase immunoprecipitation systems for measuring antibodies in autoimmune and infectious diseases. Transl Res. 2015;165:325-35.

14. Aye KM, Nagayasu E, Baba M, Yoshida A, Takashima Y, Maruyama H. Evaluation of LIPS (luciferase immunoprecipitation system) for serodiagnosis of toxoplasmosis. J Immunol Methods. 2018;462:91-100.

15. Andiappan $H$, Nissapatorn V, Sawangjaroen N, Nyunt MH, Lau YL, Khaing SL, et al. Comparative study on Toxoplasma infection between Malaysian and Myanmar pregnant women. Parasit Vectors. 2014:7:564

16. Thai TL, Jun H, Park SH, Le HG, Lee J, Ahn SK, et al. Seroprevalence of Toxoplasma gondii among school children in Pyin Oo Lwin and Naung Cho, upper Myanmar. Korean J Parasitol. 2019;57:303-8.

17. van Enter BJD, Lau YL, Ling CL, Watthanaworawit W, Sukthana Y, Lee WC et al. Seroprevalence of Toxoplasma gondii infection in refugee and migrant pregnant women along the Thailand-Myanmar border. Am J Trop Med Hyg 2017:97:232-5.

18. Wei HX, He C, Lindsay DS, Peng HJ. Relationship between cat contact and infection by Toxoplasma gondii in humans: a meta-analysis. Comp Parasitol. 2016;83:11-9.

\section{Publisher's Note}

Springer Nature remains neutral with regard to jurisdictional claims in published maps and institutional affiliations.

\section{Ready to submit your research? Choose BMC and benefit from:}

- fast, convenient online submission

- thorough peer review by experienced researchers in your field

- rapid publication on acceptance

- support for research data, including large and complex data types

- gold Open Access which fosters wider collaboration and increased citations

- maximum visibility for your research: over $100 \mathrm{M}$ website views per year

At $\mathrm{BMC}$, research is always in progress.

Learn more biomedcentral.com/submissions 